\title{
Il tormentato percorso di una biografia: Gallarati Scotti racconta Fogazzaro
}

\author{
Claudia CREVENNA \\ Università degli Studi di Milano ${ }^{1}$ \\ claudia.crevenna@unimi.it
}

Recibido: $01 / 10 / 2013$

Aceptado: 25/11/2013

\section{RIASSUNTO}

L'articolo intende analizzare le complesse fasi dell'elaborazione della Vita di Antonio Fogazzaro scritta dall'amico e discepolo Tommaso Gallarati Scotti. Presentando le difficoltà di ricostruzione, che le diverse fasi di scrittura comportano, per quanto riguarda la ricostruzione della volontà autoriale, è anche possibile definire meglio il rapporto Fogazzaro/Gallarati Scotti, e quello fra la gerarchia cattolica e due importanti esponenti del "modernismo".

Parole chiave: Antonio Fogazzaro, biografia, index librorum pribitorum, ecdotica, edizione critica.

\section{The Difficult Making of a Biography: Gallarati Scotti Tells on Fogazzaro}

\begin{abstract}
The aim of this article is to analyze the difficult making of "Vita di Antonio Fogazzaro" written by a friend and pupil of Fogazzaro himself, Tommaso Gallarati Scotti. The discussion about the textual problems, due to the changes in the different versions, and the re-construction of the true authorial figure will permit an attentive view of the relationship between Fogazzaro and Gallarati Scotti, evidencing the contrast between these two modernist intellectuals and the catholic hierarchy.
\end{abstract}

Keywords: Antonio Fogazzaro, Biography, Index librorum proibitorum, Ecdotic, Critical Edition.

SOMMARIO: 1. Introduzione 2. Le varie edizioni: analisi preliminare 2.1. Dalla prima alla seconda edizione: aggiunte e riduzioni 2.1.1. Aggiunte 2.1.2. Riduzioni 2.2. L'analisi dell'opera fogazzariana 2.2.1. La corrispondenza con Elena 2.2.2. Piccolo Mondo Antico 2.2.3. Il Santo 2.3. Altri aspetti della Vita 3. Prime conclusioni.

${ }^{1}$ Dipartimento di Studi Letterari, Filologici e Linguistici, Via Festa del Perdono 3 e 7, I20122, Milano (Mi), Italia. 


\section{INTRODUZIONE}

Quando decide di scrivere la biografia di Antonio Fogazzaro, tenendo fede all'incarico che Fogazzaro stesso gli aveva affidato ${ }^{2}$, Gallarati Scotti è spinto da motivazioni che non hanno a che vedere con l'interesse per le biografie letterarie, $o$, in generale, con l'attività di studioso e di autore di saggistica e letteratura cui pure Gallarati si era dedicato. È anzitutto una esigenza morale profonda, uno slancio d'affetto verso l'amico e maestro Fogazzaro, un bisogno di riabilitarne la fama e il valore di fronte ai suoi critici e detrattori. È il desiderio di raccontare una figura a suo modo esemplare, che Gallarati aveva conosciuto, che aveva ammirato e a cui si era legato di sincera amicizia, condividendo una parte delle sue idee e del suo percorso di autore e di pensatore, proprio quando tale percorso si fece particolarmente accidentato e doloroso.

Chiaramente ciò comporta per Gallarati la scelta di un punto di vista molto particolare che rende l'intera sua opera qualcosa di diverso dal classico genere della biografia, ottenendo un risultato particolarissimo: una sorta di biografia romanzata ${ }^{3}$. Infatti decide di raccontare Fogazzaro da un punto di vista interno, come forse Fogazzaro stesso si sarebbe raccontato. Si tratta di una scelta audace e sicuramente fuori dagli schemi, tanto da suscitare perplessità persino in ambienti vicini a Gallarati, come dimostra la recensione del marchese Filippo Crispolti, giornalista e politico, caro amico di Gallarati. Sulla "Nuova Antologia", all'indomani della pubblicazione della Vita di Antonio Fogazzaro, Crispolti rileva, al termine di una lunga ricognizione sul contenuto dell'opera, quest'unico limite nel poderoso lavoro dell'amico Gallarati Scotti, cioè l'aver visto e narrato Fogazzaro troppo "dall'interno": «lo Scotti [...] ha fatto subire in qualche modo al personaggio suo ciò che il Fogazzaro fece talvolta subire ai suoi personaggi maggiori, che cioè a forza di studiarli internamente non li rese abbastanza visibili all'esterno» (Crispolti 1920: 141).

Tuttavia il limite che Crispolti individua nel lavoro di Gallarati è di fatto la cifra più significativa dell'opera, perché l'operazione di Gallarati consiste nel proiettare se stesso nel Fogazzaro, parlare di sé parlando di lui; questo è di fatto l'osservatorio speciale e appassionato che solo Gallarati poteva avere del maestro e amico. Come ha correttamente rilevato Mattesini, «nella Vita di Antonio Fogazzaro si proietta la vita stessa di Tommaso Gallarati Scotti. In alcuni tratti della biografia interiore del

${ }^{2}$ Nella prefazione alla sua biografia, Gallarati riporta le parole che gli rivolse lo stesso Fogazzaro: «tu sei l'uomo più adatto a scrivere di me dopo la mia morte e allora avrai tutti i materiali possibili, tutta la libertà. Questo è il mio voto» (Gallarati Scotti 2011: 14). La pubblicazione del 2011 ripropone il testo della prima edizione dell'opera, pubblicata per i tipi di Baldini e Castoldi nel 1920 (tutte le citazioni del testo della Vita del 1920 sono prese dalla nuova pubblicazione del 2011).

${ }^{3}$ Non a caso Mattesini parla di «antiromanzo» e «antisaggio» (Mattesini 1994: 231). Anche Fabio Danelon descrive le caratteristiche peculiari di questa biografia che si configura «quasi come archetipo, per certi versi, d'una modalità di biografia personalmente partecipata e ideologicamente coinvolta» (Danelon 2010: 104). 
"maestro" è possibile infatti veder riflessi sentimenti e idee, memorie e sofferenze che animarono, turbarono e ferirono il "discepolo"» (Mattesini 1994: 215) ${ }^{4}$. Si tratta in sostanza di una biografia che è anche autobiografia ${ }^{5}$, in un gioco di specchi per cui il biografo si riflette nel biografato, e il biografato a sua volta si riflette nei personaggi dei suoi romanzi ${ }^{6}$. Al punto tale che lo stesso Fogazzaro diventa in un certo senso personaggio del romanzo-nonromanzo che racconta la sua vita. Forse involontariamente, Crispolti sceglie dunque un'espressione quantomai adeguata, quando parla di Fogazzaro come «personaggio» dell'opera del Gallarati, perché davvero la Vita di Antonio Fogazzaro si può collocare in una zona liminale tra la biografia, il saggio e il romanzo.

Analizzando questo aspetto dell'opera, cioè la sua tendenza ad essere, almeno in parte, una sorta di romanzo, Francesco Spera, in un saggio apparso a corredare la nuova pubblicazione della Vita di Antonio Fogazzaro, sottolinea come la descrizione della morte dell'autore vicentino, narrata con accenti commossi e ricchi di pathos da Gallarati, testimone diretto dell'agonia del maestro, sia tutta tracciata sulla falsariga della prosa fogazzariana: Gallarati Scotti, nel descrivere gli ultimi momenti di vita del Fogazzaro, si mostra «buon emulo del maestro» (Spera 2011: 467), trasfigurandolo quasi nel personaggio di uno dei suoi romanzi.

La biografia del Fogazzaro è dunque la «storia di un'anima», come Gallarati stesso dichiara apertamente nella prefazione alla prima edizione ${ }^{7}$. Una storia che si snoda a partire dall'infanzia dell'autore, ripercorrendo a mo' di proemio le vicende giovanili del padre Mariano e ricostruendo l'ambiente famigliare nel quale Fogazzaro nacque e crebbe. La Vita racconta l'educazione, l'adolescenza e la maturità del poeta, e si concentra poi, in buona parte, sulle opere letterarie, viste

${ }^{4}$ Già Gentile, nella recensione comparsa all'uscita della Vita nel '20 aveva individuato questo aspetto fondamentale della biografia come autobiografia, con i pregi e i limiti che ciò comporta: «un libro dunque che ha più dell'autobiografia che della vera e propria biografia; la quale è sempre storia, e perciò giudizio, che importa superamento e distacco dell'autore dalla sua materia. Dell'autobiografia ha - e questo è il suo grandissimo pregio - la ricchezza e sicurezza di intuizione interna. [...] Carattere autobiografico, reso possibile [...] sopra tutto, dalla simpatia intellettuale e comunanza d'idee onde [Gallarati] era stato a lui legato per vari anni [...]. E allora la storia del Fogazzaro fu pure la storia del G.-S. [...] e la biografia in questo punto [...] si trasforma naturalmente in autobiografia» (Gentile 1920: 291-292).

${ }^{5}$ Sempre Mattesini spiega che Tommaso Gallarati Scotti «con la sua biografia del 1920, inaugura, in effetti, un esempio di scrittura anche strutturalmente assai distinta e complessa [...] ove appunto la storia compare mista all'invenzione senza che questa rimanga soffocata dal copioso materiale raccolto e utilizzato. Biografia anzitutto - direi - come autobiografia» (Mattesini 1994: 230).

${ }^{6}$ Con precisione Francesco Spera (2011) ha analizzato il meccanismo di presentazione delle opere di Fogazzaro da parte di Tommaso Gallarati Scotti, mostrando come l'analisi dei personaggi sia sempre volta a mettere in luce aspetti della personalità dell'autore, che i personaggi in qualche modo incarnerebbero.

${ }^{7}$ «Io ho desiderato di scrivere la storia della sua anima» (Gallarati Scotti 2011: 14). 
come altrettante tappe del cammino interiore dell'animo di Fogazzaro, sino alla morte $^{8}$. Per questo tanto si insiste, secondo uno schema che si ripete per ogni romanzo, su un'attenta disamina della psicologia dei protagonisti dei romanzi fogazzariani, come se in ognuno di essi si trovasse una parte dell'animo del loro autore, uno slancio ideale, un turbamento, una passione da esorcizzare o una meta da raggiungere. Di preferenza nominato «il poeta», Fogazzaro non è inquadrato tanto o solo come letterato, sebbene la lettura e l'analisi dei romanzi sia il cuore della sua biografia, ma piuttosto come pensatore cattolico e liberale, che ha contribuito alla rinascita culturale dell'Italia post-unitaria ${ }^{9}$. Anche laddove insiste sul valore letterario dell'autore, Gallarati lo fa sulla scia di quegli uomini di lettere che furono anzitutto uomini di pensiero e impegno politico e morale, come Dante ${ }^{10}$. Da questa prospettiva si può pensare di interpretare anche lo scarso rilievo dato da Gallarati ai rapporti del Fogazzaro con i letterati del suo tempo. Nella sua analisi di come Gallarati vede e descrive il Fogazzaro, Gianni Eugenio Viola ritiene che tale silenzio circa i contatti tra Fogazzaro e il mondo letterario a lui contemporaneo, un silenzio che riguarda non solo la Vita, ma anche la pubblicazione, nel 1940, delle Lettere scelte dell'epistolario di Fogazzaro (Fogazzaro 1940) sia studiatamente calcolato da parte di Gallarati; e tuttavia si può pensare che lo scopo di Gallarati non sia forse di voler dare l'impressione di un Fogazzaro minore ${ }^{11}$, ma semmai di porre l'autore fuori del suo tempo, erede idealmente di Dante prima ancora che in rapporto dialettico con un Verga o - questione ancora più spinosa - con un Manzoni ${ }^{12}$. Non si tratta di un'operazione dunque riduttiva della figura del

${ }^{8}$ Madrignani, nell'introduzione all'edizione della Vita del 1982, arriva a dire che «la prospettiva in cui ha lavorato l'autore è quella di farci balenare l'itinerarium ad Deum di questo maestro di santa laicità» (Madrignani 1982: X).

9 «Si può affermare che questa è la tesi centrale della Vita, la proposta di un'interpretazione che non è soltanto letteraria, ma culturale in senso lato, con la rivendicazione del ruolo che i cattolici hanno svolto nel Risorgimento contribuendo in modo determinante alla rinascita "liberale e nazionale"» (Spera 2011: 462).

${ }^{10}$ Non a caso Gallarati pubblica nel 1921 la Vita di Dante, seguendo lo stesso schema della biografia interiore o biografia romanzata, tracciata ripercorrendo le opere principali dell'autore. La Vita di Dante, varie volte rimaneggiata, è stata ristampata nella sua prima versione nel 2009, per Franco Angeli, a cura di Francesco Spera.

${ }^{11}$ Circa la scelta delle lettere pubblicate nell'epistolario, così Viola commenta: «appena trentasei destinatari, per lo più rappresentati da pochi fogli; non quindi certo l'epistolario dell'autore di notorietà internazionale al quale il giovane Gallarati Scotti s'era avvicinato. E questa sensazione di una scelta di modestia, di profilo quasi da marginale nelle lettere nostre si conferma dal raffronto tra i due testi, la biografia e l'epistolario». E continua, commentando le lettere scelte per la pubblicazione, precisando che tra esse: «non v'è traccia di un carteggio col De Amicis (che pur dovette esserci [...]). Né una sola lettera che riguardi i rapporti di edizione; condizione curiosa certo trattandosi dell'epistolario (e della biografia) d'un grande scrittore» (Viola 1994: 369).

12 Sempre Viola: «Gallarati Scotti non seppe leggere il Fogazzaro nell'ottica storiografica che forse l'autore chiedeva, e che l'avrebbe visto posto accanto al Verga ed al D’Annunzio» (Viola 1994: 383). 
Fogazzaro, quanto piuttosto di una sua esaltazione quale uomo di lettere pronto a mettere la sua penna al servizio di ideali che vanno ben oltre la letteratura e dunque ben oltre la contingenza del rapporto con altri letterati ${ }^{13}$, sebbene il dibattito religioso e politico contemporaneo lo vedesse invece completamente e appassionatamente coinvolto, considerando il percorso accidentato della sua produzione letteraria, soprattutto a partire dal romanzo Il Santo, sottoposto all'occhiuto controllo della censura ecclesiastica che infatti ne determinò la messa all'Indice.

Il rapporto conflittuale con l'autorità ecclesiastica, imperniato sul movimento modernista, costituisce uno dei nodi più significativi e dolorosi della biografia, sia perché effettivamente la tensione religiosa fu al cuore del pensiero e della produzione di Fogazzaro, sia perché il dibattito sul Modernismo coinvolse non solo Fogazzaro ma anche il suo biografo ${ }^{14}$. Tanto più che l'elaborazione del Santo coincide, indicativamente, con il periodo in cui si strinsero i rapporti di amicizia tra Gallarati stesso e Fogazzaro, con il risultato che Gallarati entra in scena a sua volta nella Vita come "personaggio" e testimone diretto delle vicende nelle quali, e lo ammette egli per primo ${ }^{15}$, risulta inevitabilmente coinvolto ${ }^{16}$.

La messa all'Indice della Vita nel 1920 fu il motivo principale che spinse Gallarati a una revisione del testo, affinché potesse essere nuovamente pubblicato con il benestare dell'autorità ecclesiastica. Con l'aiuto del cardinale Pietro Maffi prima e soprattutto poi, morto il Maffi nel 1930, del domenicano Mariano Cordovani, Gallarati provvide a una revisione del testo. Del resto, avute avvisaglie delle perplessità con cui veniva accolta in ambito ecclesiastico la Vita, Gallarati si era immediatamente dichiarato pronto ad emendarla per non incorrere nella condanna; tuttavia la situazione precipitò rapidamente e, nonostante gli iniziali ottimismi dell'amico Crispolti, fiducioso in qualche rassicurazione avuta dall'alto

${ }^{13}$ Nota Madrignani, nella sua introduzione: «i romanzi [di Fogazzaro] non sono mai messi a confronto con la produzione letteraria del tempo, perché il biografo li considera o sull'onda lunga della civiltà letteraria del cattolicesimo italiano oppure all'interno della vicenda psichica dello scrittore, fuori da ogni considerazione storico-politica e di ogni condizionamento materiale» (Madrignani 1982: IX-X).

${ }^{14}$ A proposito della formazione di Gallarati e dei suoi contatti culturali, intellettuali e religiosi, si vedano le pagine di Maurilio Guasco (2011) e il profilo tracciato da Nicola Raponi (1972).

${ }^{15}$ «Io sento, toccando di questo momento della vita di Antonio Fogazzaro, la difficoltà di trattarne con quella serenità che una falsa concezione dell'oggettività storica confonderebbe quasi con l'indifferenza [...]. Per poter essere indifferente io non dovrei sentirmi in alcun modo partecipe alla lotta religiosa di quel periodo. Dovrei aver rinnegato il mio passato o sentirlo morto in me. Ora questo non è. Sento vive le radici che mi legano a quell'ora di lotta» (Gallarati Scotti 2011: 284).

16 «In particolare il coinvolgimento dell'autore emerge subito quando si comincia a manifestare la nuova volontà di Fogazzaro di utilizzare il genere del romanzo per una missione definita espressamente "religiosa"» (Spera 2011: 464). 
che pareva scongiurare l'ipotesi di una condanna all'Indice ${ }^{17}$, il meccanismo era ormai innescato e, a seguito anche di alcune recensioni piuttosto critiche ${ }^{18}$ sull'opera, il 9 dicembre 1920 la condanna arrivò ${ }^{19}$.

Il successivo lavoro di risistemazione del testo portò dunque all'edizione del 1934, presso Mondadori, con un titolo significativamente ampliato: La vita di Antonio Fogazzaro. Dalle memorie e dai carteggi inediti. La nuova formulazione del titolo tiene conto del parallelo lavoro di sistemazione dell'epistolario del Fogazzaro, che aveva permesso a Gallarati di entrare in possesso di materiale documentario nuovo, di cui si giovò anche per ampliare la sua biografia. Ci sarà poi un'ulteriore edizione nel '63, sempre per Mondadori, che Gallarati dovette ritenere definitiva $^{20}$. A proposito del passaggio da un'edizione all'altra, scrive Fabio Danelon: «benché sia certo auspicabile un'edizione critica che collazioni le differenti edizioni (compresa l'ultima del 1963), l'impressione che si ricava è che l'edizione del 1934 non differisca profondamente da quella del 1920» (Danelon 2010: 102). L'impressione di Danelon è senz'altro corretta. La recente edizione della Vita di Antonio Fogazzaro, uscita per l'editrice Morcelliana nel 2011, ripropone l'edizione del 1920, dunque la prima, considerandola come quella che risponde «maggiormente al pensiero e agli intenti dell'autore» ${ }^{21}$. Questa pubblicazione, promossa dal Centro Studi Tommaso Gallarati Scotti, si è giovata di un preliminare lavoro di collazione, che ha permesso di individuare gli interventi successivi di Gallarati dalla prima all'ultima edizione e del quale si vuole qui

17 «Il Papa disse formalmente che se anche verranno denunzie in piena regola e si facciano insistenze per una condanna, questa sarà da Lui rifiutata perché bisogna tener conto della buona volontà d'emendare» (Lettera di Filippo Crispolti a Tommaso Gallarati Scotti, 2 luglio 1920, riportata in appendice al saggio di Marcora 1984: 314)

${ }_{18} \mathrm{Si}$ veda soprattutto la recensione del gesuita Enrico Rosa, che ebbe, su incarico del Sant'Uffizio, una parte non indifferente nel meccanismo di condanna all'Indice. Su $L a$ Civiltà Cattolica, il Rosa scriveva: «non vediamo dunque come, senza gran pericolo, si possa proporre a modello, e perciò senza riserva si possa lodare chi volendo tesserne la storia, ne fa praticamente, anche proponendosi e protestando il contrario, e anche in punti i più delicati, che toccano la fede e la disciplina cattolica, ne fa l'apologia, l'esaltazione, la quale riesce infine ad una tentata difesa dei suoi metodi e delle sue dottrine, come ad una tacita condanna della Chiesa che le ha riprovate». E ancora, ben cogliendo la condizione ibrida dell'opera tra biografia, saggio e romanzo, aggiunge: «Certo, vorremmo anche noi distenderci piacevolmente nella critica letteraria [...]; né vorremmo allora risparmiare, in questa parte, le lodi al suo biografo, che mostra, egli pure, belle doti di letterato e di critico. [...] ma con che frutto? Forse con questo solo: che [...] nasconderebbero il pericolo o la reità del veleno occulto tra i fiori della letteratura» (Rosa 1920: 68-69).

${ }_{19}$ Per una ricognizione precisa e documentata delle vicende che determinarono la condanna all'Indice della Vita, si veda il saggio di Carlo Marcora (1984), e, più recentemente, il saggio di Annibale Zambarbieri (2013).

${ }^{20}$ L'edizione dell'82, sempre per Mondadori, con l'introduzione di Carlo Alberto Madrignani, ripropone il testo del ' 63.

${ }^{21}$ Così si legge nell’ «Avvertenza» all’edizione della Vita del 2011. 
proporre qualche campione significativo. I risultati della collazione sono tra l'altro meglio interpretabili alla luce della consultazione del manoscritto della Vita, conservato presso il Centro Studi Gallarati Scotti ${ }^{22}$.

\section{LE VARIE EDIZIONI: ANALISI PRELIMINARE}

Anzitutto si deve rilevare che la considerazione di Danelon coglie nel segno. I cambiamenti tra le varie edizioni, molto più significativi tra la prima e la seconda edizione, mentre per lo più minuti e spesso solo stilistici tra la seconda e la terza, mostrano una volontà di limare e aggiustare le parti più spigolose e puntute della Vita, quelle parti che verosimilmente determinarono la condanna all'Indice ${ }^{23}$. Sono pertanto cambiamenti significativi, ma non intaccano l'impianto generale dell'opera. Il sapiente gioco di equilibrismi, come ha correttamente colto Madrignani $^{24}$, di cui Gallarati ha dato prova nel passaggio dalla prima alla seconda edizione, permette alla fine di ottenere un testo accettabile dal punto di vista della dottrina ecclesiastica e tuttavia ancora palpitante di quelle posizioni e idee che, più scopertamente presenti nell'edizione del 1920, si colgono più che altro per reticenza nell'edizione del ' 34 . In ciò in fondo Gallarati si dimostra capace di prudenza ma non di tradimento, e, pur rientrando nell'alveo di posizioni ritenute ortodosse, riesce a non smentirsi. Sotto questo aspetto non si può dire che non avesse colto nel vero Enrico Rosa, quando, a seguito della sua puntigliosa analisi del testo, aveva di fatto concluso: «che il libro era non solo condannabile, ma "da proscriversi il più prontamente [...]". Né servivano aggiustamenti in vari punti, ma si imponeva la decisione di rifarlo da capo: non dunque una ristampa o un'edizione corretta, ma un'opera nuova» (Zambarbieri 2013: 28) ${ }^{25}$. Il rifacimento dunque del testo

${ }^{22}$ Vorrei qui ringraziare il Principe Fulco Gallarati Scotti, nipote di Tommaso, e il Presidente del Centro Studi Gallarati Scotti, Luciano Pazzaglia, che mi hanno permesso la consultazione del manoscritto. Desidero inoltre ricordare Nino Del Bianco, che fu amico di Gallarati, per il suo aiuto nell'analisi delle carte manoscritte conservate presso il Centro.

${ }^{23}$ Anche se effettivamente «la biografia di Fogazzaro non sembra contenere gravi errori dottrinali» (Guasco 2011: 450).

${ }^{24}$ «Non si vuol con questo affermare che gli interventi correttivi siano poco significativi, specie in certe parti e segnatamente negli ultimi capitoli dove la questione modernista ha un peso determinante; ma si tratta di correzioni non essenziali, per così dire di "aggiustature", fatte con calcolata abilità in vista di un compromesso onorevole fra il già scritto e la incalzante minaccia ecclesiastica» (Madrignani 1982: VII).

${ }^{25}$ Più estesamente, nel Voto di padre Enrico Rosa che Zambarbieri riporta in appendice al suo saggio si legge: «ho udito da varie parti che l'autore è pronto a correggere e desidera sapere quali siano le proposizioni dannabili; con questo egli vorrebbe evitare la condanna, promettendo una nuova edizione corretta. Ma in ciò vi è, secondo me, un danno e un pericolo: il danno, che la edizione presente resterebbe senza la condanna che si merita, e perciò con un'autorità che sarebbe fonte di errore; il pericolo, in quanto la nuova edizione, pure rimanendo sostanzialmente qual è, un'apologia del modernista, solo perché si direbbe 
nell' edizione del '34, perfezionato, limato, aggiustato in numerosissimi punti, comportò alla fine l'accettazione da parte dell'autorità ecclesiastica, anche se, a ben vedere, si tratta di un rifacimento basato più sulla prudenza che su un effettivo ripensamento dell'intero lavoro. Ciò non toglie che i cambiamenti siano "di sostanza" e non solo di forma, ma se non altro non si può dire che le posizioni del Gallarati della prima edizione siano smentite nel segno di un giudizio totalmente mutato su Fogazzaro.

L'analisi del manoscritto ${ }^{26}$ infine permette di fare luce su un aspetto nuovo: molti brani che compaiono nell'edizione del ' 34 (e non in quella del '20) erano già presenti in origine nel manoscritto, ma erano stati inizialmente scartati. Nella Vita del'20, che voleva configurare come «storia di un'anima», Gallarati presumibilmente aveva deciso di espungere alcuni brani ritenuti accessori (soprattutto legati alla formazione di Fogazzaro come uomo e letterato, o alla descrizione delle trame dei romanzi), per prediligere una prospettiva tutta interiore. Invece nel ' 34 molti di questi brani ritornano, a volte esattamente come erano stati formulati all'inizio, a volte con lievi modifiche. Il reintegro di queste parti è dovuto forse proprio al tentativo di ripararsi dalle critiche, mosse anche da ambienti amici (si ricordi il Crispolti), di aver descritto il suo protagonista troppo da dentro e poco da fuori, o anche alla necessità di dare più corpo a certe parti del testo che erano state soggette a sfrondamenti resi necessari per scongiurare una nuova censura ${ }^{27}$, o ancora al bisogno di venire incontro alle esigenze di un pubblico nuovo, non più contemporaneo del Fogazzaro e dei suoi romanzi, e quindi bisognoso di maggiori ragguagli circa i contenuti delle opere dell'autore vicentino. Al recupero di parti già presenti nel manoscritto si aggiunge, naturalmente, l'inserimento di brani ex novo, dovuti forse anche alla disponibilità di nuovo materiale documentario su cui Gallarati stava lavorando parallelamente per la pubblicazione dell'epistolario di

corretta da alcune proposizioni più grossamente erronee od ereticali, si stimerebbe innocua, forse anche commendevole [...]. Non basta, secondo me, la correzione di alcuni passi: bisogna rimaneggiare il libro, in tutti i punti, dico, che non toccano questioni di critica letteraria o indifferenti alla religione» (Zambarbieri 2013: 40).

${ }^{26}$ Per una descrizione delle caratteristiche del manoscritto conservato presso il Centro Studi Gallarati Scotti si veda la mia «Nota alle tre edizioni della Vita» (Crevenna 2011: 471-473).

${ }^{27}$ Si può forse capire il retroscena della dichiarazione di Gallarati, all'indomani della pubblicazione della prima edizione, di essere subito pronto a correggere e sistemare il testo per un'edizione successiva che avesse il beneplacito dell'autorità ecclesiastica. In considerazione di tutto il materiale espunto dalla prima edizione, ma già pronto, Gallarati probabilmente sapeva bene di avere a disposizione, già predisposti, molti "pezzi di ricambio" con i quali sostituire le parti del suo testo che si fossero ritenute sconvenienti. Si veda in proposito la lettera del 15 dicembre 1920 di Gallarati al Cardinal Ferrari, che gli aveva notificato la messa all'Indice dell'opera: «V.E. sa come durante la primavera scorsa, avendo avuto conoscenza di alcune autorevoli pressioni esercitate perché la Vita di A. Fogazzaro fosse messa all'Indice, io facessi la spontanea offerta di una revisione del volume in una seconda edizione se mi fossero stati indicati specifici errori che nel libro, contro la mia volontà, avessero suonato offesa ai dogmi o alla morale cattolica» (Marcora 1984: 318). 
Fogazzaro. Dunque bisogna dare atto all'onestà intellettuale di Gallarati che non si smentisce, non si tradisce e non si rinnega, ma attinge alla sua prima produzione come si attingerebbe da un bacino di memorie, di immagini, tutte prese dal vivo dei ricordi e dei convincimenti di una vita, e quindi tutte vere, ma delle quali, secondo le necessità, è necessario fare una scelta che privilegi certi aspetti e ne taccia altri. Gallarati pertanto compie, nel rielaborare la biografia di Fogazzaro, anzitutto un'operazione di critica letteraria su se stesso e sulla propria produzione, a dimostrazione, una volta di più, di quanto la biografia di Fogazzaro sia per certi versi anche autobiografia ${ }^{28}$.

\subsection{Dalla prima alla seconda edizione: aggiunte e riduzioni}

\subsubsection{Aggiunte}

Gli interventi nel passaggio dalla prima alla seconda edizione si possono ricondurre a tre grandi aree di interesse: informazioni biografiche su Fogazzaro; valutazione delle sue opere letterarie; rapporto tra Fogazzaro e la Chiesa. Le integrazioni sulla biografia di Fogazzaro spesso hanno, oltre all'esito evidente di far conoscere più da vicino lo scrittore vicentino, anche quello di esaltarne la dirittura morale e difendere il suo pensiero dagli attacchi (quando non indebiti, quantomeno eccessivi, secondo Gallarati Scotti) che ebbe a subire. Lo stesso vale per il giudizio sulle opere letterarie, giudizio che non solo corrisponde alla lucidità di uno sguardo critico più distaccato, ma soprattutto tende a minimizzare il valore di opere discusse come Il Santo, che maggiormente acuirono il dissidio tra Fogazzaro e le gerarchie ecclesiastiche, esaltandone invece altre. Proprio quest'ultimo aspetto prevede i ripensamenti e le risistemazioni più profonde di Gallarati Scotti. Se infatti per le informazioni sul Fogazzaro uomo e letterato le correzioni si servono spesso del recupero di brani già scritti per la prima edizione, ma poi scartati (come mostra il manoscritto), per il più complesso aspetto del rapporto tra Fogazzaro e la Chiesa le modifiche apportate da Gallarati Scotti sono pensate più che altro in seguito alla messa all'Indice. Non è dunque un caso che i brani espunti nel manoscritto e poi recuperati nel '34 si concentrino soprattutto nei capitoli dell'opera precedenti al sedicesimo, La vigilia. Da questo capitolo, che comincia a trattare dell'elaborazione del Santo, in avanti, non vi sono più recuperi di parti già presenti nel manoscritto, ma tutti i cambiamenti sono ex novo. È questa con ogni evidenza la parte dove Gallarati fu costretto ad incidere con più energia nel vivo del suo testo, e rispetto

\footnotetext{
${ }^{28} \mathrm{Mi}$ permetto di rimandare alle mie considerazioni sul manoscritto della Vita: «il valore del manoscritto è pertanto decisivo sia da un punto di vista filologico sia da un punto di vista critico, perché molti passi che si sarebbero potuti credere inserimenti ex novo pensati per la seconda edizione del testo, sono invece recuperi [...] di brani già scritti in origine. E in effetti la cronistoria di questo volume è da pensarsi non come uno sviluppo in linea retta e un progressivo accrescimento di materiale, quanto piuttosto uno svolgimento a spirale, che porta a modifiche, recuperi, ripensamenti» (Crevenna 2011: 473).
} 
alla quale gli venne chiesto, pena probabilmente una nuova esclusione dall' ortodossia, di rendere le sue posizioni più sfumate.

Il primo aspetto delle modifiche comporta un arricchimento biografico della figura di Fogazzaro con lettere e aneddoti, che servono a dare, anche al lettore che non aveva condiviso lo stesso clima e lo stesso momento storico, un'immagine dell'autore dai contorni più nitidii ${ }^{29}$. Ciò si deve da un lato al nuovo materiale di cui Gallarati venne in possesso, come egli stesso dichiara, dall'altro all'esigenza di dare forse un'immagine più viva, più piena, insomma più intera dello scrittore ${ }^{30}$.

Si pensi per esempio alla lettera inviata da Fogazzaro alla suocera appena dopo il matrimonio: nel '34 essa viene riportata per esteso, mentre nell'edizione del '20 è solo citata in nota e riassunta in tre righe. La lettera raggiunge il doppio scopo di dare un grazioso momento aneddotico dei primi giorni del matrimonio di Fogazzaro, e di consacrare le sue premure di marito innamorato e felice:

[...] Partiti da Padova fra i primi soldati che precedevano l'arrivo del Re, ebbimo la ventura d'incontrare Vittorio che in calesse stava per fare il suo ingresso a Padova. L'abbiamo salutato con un "viva il Re" cui S.M. cortesemente rispose con un cenno del capo. Passato Monselice e facendosi sentire tanto alla Rita che a me alcuni sintomi d'appetito abbiamo svaligiata la pietosa e ricca sporta da lei con tanta previdenza ammannitaci...

La Rita sta benissimo ed il suo umore migliora a vista d'occhio. Cara Mamma, caro Papà, permettete che io dica con un abbraccio quanto sia la felicità che vi debbo e vi

${ }^{29}$ Gallarati stesso è profondamente convinto dell'esigenza di chiarire il profilo umano di Fogazzaro per delineare meglio l'artista, sul presupposto della connessione intima tra arte e vita: «l'aver dovuto in questo libro indagare la complessa e ricca personalità dell'artista [...] può aver dato l'impressione che io dimenticassi il lato più esemplare e domestico della sua esistenza. [...] il ritratto che ho tracciato di lui non sarebbe completo ed evidente, se non rivivessi con la sua figura scomparsa, in quella intimità dell'uomo e del cristiano, del padre di famiglia e dell'amico, per cui le ore passate insieme restano tra le migliori della mia vita giovanile. Per avvicinare oggi, a chi non l'ha conosciuto, questa sua vita famigliare mi è caro di rivivere con lui nei luoghi che amava» (Gallarati Scotti 1934: 382-383).

30 Circa la nuova disponibilità di fonti documentarie, Gallarati nella prefazione all'edizione del '34 è esplicito: «a tutto questo materiale se ne è venuto aggiungendo di mano in mano anche dopo la pubblicazione della prima edizione della Vita [...]. Tale tesoro [...], accuratamente scelto nel volume delle Lettere che è in corso di pubblicazione presso la Casa Mondadori e che dovrà raccogliere il fiore degli scritti più intimi del Fogazzaro, ci ha servito per illuminare di luce nuova la sua figura di uomo e di scrittore» (Gallarati Scotti 1934: XIII). Quanto invece alla volontà di dare del Fogazzaro un'immagine più viva, si pensi ad esempio alla frase, inserita nell'edizione del '34 e assente nel '20, che commenta la narrazione drammatica della morte del figlio Mariano: «sentendolo soffrire noi lo conosceremo meglio nella sua più intima grandezza d'uomo. Perché è di fronte a suo figlio morto che noi scopriamo in lui dietro il romanziere, dietro il poeta, dietro il pensatore già celebre, qualcosa di assai più elementare ma più grande che ci tocca e ci avvicina a lui con una simpatia intera: noi troviamo il padre, che amava il figlio assai più che la gloria» (Gallarati Scotti 1934: 265). 
rinnovi le ardenti promesse che v'ho già fatto di consacrarmi tutto a questo vostro gioiello che avete voluto confidarmi. (Gallarati Scotti 1934: 37)

Segue a questa una nuova lettera, questa volta della madre di Fogazzaro, citata per intero nel ' 34 e del tutto assente nel ' 20 :

E dalla diletta Valsolda i genitori sognavano intanto ansiosi i primi passi sulla via della tanto desiderata felicità del figlio. Basti, tra le molte, questa toccante lettera della madre in cui è il timbro dei soavissimi affetti famigliari che circondavano il giovane poeta sposo. "Miei carissimi. Questa piccola colonia di Oria vi seguita come il suo Pianeta il satellite. Mi compiaccio pensando che sarete arrivati a Parma vispi e felici come due passeri. Tutto vi parrà bello e simpatico. Portate questa vostra disposizione anche ad Oria e questo caro paesetto esulterà di ricevervi. Il papà sta studiando col passeto tutti i vani della vostra cameretta e si affanna della ristrettezza dello spazio e vorrebbe portare in là con gli occhi e col fiato i confini delle pareti purché ci poteste stare senza disagio. Ma tant'è, tutto è piccolo e bisogna starci là, confidiamo nella bontà della Rita e nell'amore che non disprezza i nidi piccoli”. (Gallarati-Scotti 1934: 37-38)

O ancora, per sottolineare la sintonia di Fogazzaro con la natura, la sua capacità di penetrare il suo segreto linguaggio, Gallarati nel ' 34 aggiunge il brano suggestivo di una affettuosa lettera di papà Mariano Fogazzaro al figlio Antonio, nella quale il padre si compiace delle cure amorevoli riservate dal figlio alle piante del giardino: «mio caro ortolano... Mi sono consolato per il povero trovatello del mio giardinetto che la tua mano caritatevole abbia tolto a ravviargli indosso la vesticciuola e l'idea di avere un successore in questo pietoso ufficio mi ha tranquillato sulle sorti future del tapinello» (Gallarati Scotti 1934: 62).

Altri inserimenti, più minuti, servono a dare un'idea di ambiente, qualche pennellata di colore, come a proposito di casa Venini, a Varenna, frequentata dal giovane Fogazzaro dove, si aggiunge nel '34, «si faceva musica da mattina a sera, con serenate al chiar di luna dirette da quello spirito bizzarro che fu il celebre violoncellista Gaetano Braga» (Gallarati Scotti 1934: 32).

Inoltre, alcune precisazioni dell'edizione del ' 34 hanno lo scopo di documentare con fonti autorevoli gli episodi narrati: è quanto accade a proposito del mondo milanese che Fogazzaro frequentò durante il suo soggiorno a Milano; il racconto degli ambienti, dei personaggi, dei contatti che ebbe il Fogazzaro è chiosato nell'edizione del '34 da una piccola ma significativa nota a piè pagina che manca invece nell'edizione del '20: "gran parte di queste notizie sul mondo milanese della "scapigliatura" e i suoi rapporti col Fogazzaro mi fu trasmessa da Arrigo Boito stesso» (Gallarati Scotti 1934: 44). È chiaro che anche nel '34 Gallarati non abdica al suo ruolo di testimone diretto di molti degli eventi narrati (la frequenza dell'uso della prima persona lo dimostra) e tuttavia tiene a dare un valore documentario più circostanziato e più autorevole possibile agli episodi descritti. 


\subsubsection{Riduzioni}

Quanto detto sinora riguarda le aggiunte: il mondo famigliare e quotidiano degli affetti del Fogazzaro nell'edizione del '34 risulta arricchito di ulteriori pennellate. Ma vi sono anche importanti interventi di riduzione: nel ' 34 si smussano alcune valutazioni un po' azzardate che avrebbero forse potuto insospettire nuovamente l'autorità ecclesiastica. Sono piccoli aspetti, spesso singoli sintagmi, che mostrano con quanta meticolosità Gallarati sia intervenuto sul suo testo. Ecco uno specchietto con due piccoli esempi di alcune di queste modifiche (in grassetto le parti che subiscono cambiamenti).

\begin{tabular}{|c|c|}
\hline Edizione del '20 & Edizione del '34 \\
\hline \multicolumn{2}{|c|}{ CAP. I } \\
\hline $\begin{array}{l}\text { il primo insegnamento religioso che ebbe il } \\
\text { piccolo Antonio non fu di formule } \\
\text { dogmatiche, ma di esempi vissuti }\end{array}$ & $\begin{array}{l}\text { il primo insegnamento religioso che ebbe il } \\
\text { piccolo Antonio fu di esempi vissuti }\end{array}$ \\
\hline $\begin{array}{l}\text { se la raffica batteva contro le cadenti difese } \\
\text { del temporalismo, obbligando la Chiesa a } \\
\text { ritirarsi gradatamente dalla sua base di } \\
\text { potenza terrestre; la monaca mistica } \\
\text { scopriva anche in questo incalzare di } \\
\text { avvenimenti, temuti dalle anime timide, } \\
\text { la volontà di Dio liberatore e ricreatore } \\
\text { che rinnovava la società religiosa per } \\
\text { mano dei suoi stessi nemici. }\end{array}$ & $\begin{array}{l}\text { se la raffica batteva contro le cadenti difese } \\
\text { del temporalismo, la monaca mistica } \\
\text { scopriva anche in questo incalzare di } \\
\text { avvenimenti la volontà di Dio. }\end{array}$ \\
\hline \multicolumn{2}{|c|}{ CAP. III } \\
\hline $\begin{array}{l}\text { Col matrimonio egli aveva dovuto tornare } \\
\text { alla vita esteriore della Chiesa, quantunque } \\
\text { la sua anima ne rimanesse lontana. Vi è } \\
\text { infatti un modo puramente formale di } \\
\text { partecipare al cattolicismo che non } \\
\text { implica nessuna reale adesione alla sua } \\
\text { dottrina o alla sua morale. Per } \\
\text { moltissimi il rito domestico si concilia con } \\
\text { la fede morta e le sopravvive. }\end{array}$ & $\begin{array}{l}\text { Col matrimonio egli aveva dovuto tornare } \\
\text { alla vita esteriore della Chiesa, quantunque } \\
\text { la sua anima ne rimanesse assente. Per } \\
\text { alcuni, purtroppo, il rito domestico si } \\
\text { concilia con la fede morta e le sopravvive. }\end{array}$ \\
\hline
\end{tabular}

Nel primo esempio è evidente il tentativo di evitare una sfumatura negativa associata al dogmatismo della catechesi, un aspetto che per altro lo stesso padre Rosa aveva con meticolosità sottolineato nel documento che compilò su incarico del Sant'Uffizio, indicando questa espressione come male sonans ${ }^{31}$. Anche il terzo esempio va nella stessa direzione: i formalismi furono uno degli aspetti più avversati dal movimento modernista ed effettivamente la formulazione del '20

31 «"Male sonans", alludendo al catechismo contro il cui insegnamento accumularono tante accuse i modernisti» (si veda l'appendice con il Voto del padre Rosa (Zambarbieri2013:34). 
avrebbe potuto destare più di una perplessità negli ambienti ortodossi ${ }^{32}$. Per questo viene cautamente eliminata. Il secondo brano (nel quale la «monaca» cui si fa riferimento è la zia di Fogazzaro, suor Maria Innocente) è un esempio perfetto di attenta limatura tesa ad evitare giudizi inopportuni sugli errori della Chiesa o lusinghieri verso «i suoi stessi nemici», mantenendo invece intatta la figura quasi eroica della «monaca mistica», che ebbe la sua parte di influenza sul giovane Antonio.

\subsection{L'analisi dell'opera fogazzariana}

Dal capitolo terzo si comincia l'analisi delle opere letterarie, che prosegue sostanzialmente sino alla fine della Vita, intercalata da momenti in cui ci si torna a concentrare su episodi particolarmente significativi della biografia di Fogazzaro. L'attenzione alle opere non è la mera valutazione di un prodotto letterario, circoscritto rispetto alla totalità della vita e del pensiero del suo autore, ma è un giudizio che inevitabilmente coinvolge l'autore reale nelle sue convinzioni più profonde, perché - sostiene Gallarati - il suo intento è di studiare la vita e l'arte «non come cose artificiosamente staccate, ma come lati di una inscindibile unità» (Gallarati Scotti 2011: 64). Nel passaggio da un'edizione all'altra rimane l'idea che Piccolo Mondo Antico sia il vertice letterario toccato da Fogazzaro, tuttavia vi è una correzione del giudizio critico a proposito delle altre opere, così che nel ' 34 si tende sostanzialmente a sminuire la portata del Santo, o comunque a darne una lettura per quanto possibile più letteraria e meno attenta agli aspetti impegnati del testo sul versante religioso (operazione di difficile equilibrismo per Gallarati, se si pensa all'assunto di fondo che le opere del Fogazzaro sono opere intellettualmente e culturalmente militanti). Sulle opere letterarie si concentrano anche le lievi modifiche che Gallarati apporta nell'edizione del '63, un'ultima edizione che non aveva più l'urgenza di adeguarsi all'ortodossia e porsi al riparo dagli strali della censura, e che pertanto può dedicarsi a un lavoro più che altro di cesello, di stile, oltre che di attento bilanciamento del giudizio critico sulle opere del Fogazzaro con un distacco, foss'anche solo cronologico, che determina qualche aggiustamento e qualche ritocco nella valutazione complessiva delle opere, come si vedrà.

Esigenza generale, nel passaggio dalla prima alle edizioni successive, è far rientrare a pieno titolo Fogazzaro nel canone dei grandi autori della letteratura

${ }^{32}$ A proposito dell'espressione precedente, aggiungeva padre Rosa «non insisterei su questa proposizione, se fosse scritta da un autore non sospetto» (Zambarbieri 2013: 34). Il problema dunque, e Gallarati ne era consapevole, non era legato a singole espressioni, ma all'insieme di tante affermazioni e giudizi lasciati cadere in più punti nel testo, sommati, quel che è peggio, al fatto che egli poteva considerarsi tutt'altro che «autore non sospetto», se si considera che nel 1907 il periodico Il Rinnovamento, del quale Gallarati era stato cofondatore, venne colpito dagli strali della censura ecclesiastica in quanto accusato di Modernismo, e la stessa sorte toccò nel 1912 ai suoi racconti dal titolo Storie dell'amore sacro e dell'amore profano. 
italiana e, a distanza di tempo, si rende probabilmente necessario per Gallarati puntualizzare il valore del contributo che Fogazzaro diede come intellettuale impegnato non solo alle lettere italiane ma, per certo verso, alla crescita sociale e politica del proprio paese. Non a caso il capitolo quarto, «La vocazione per il romanzo», vede nell'edizione del '34 l'inserimento di un piccolo brano dal tono sostenuto e potente, assente nell' edizione del '20:

Nella pienezza delle sue forze e giunto ormai a quell'età in cui l'uomo sente con desidero e con sgomento insieme che le ore decisive per il proprio destino sono imminenti, si preparava a camminare con costanza per un sentiero che egli sentiva il suo, verso una meta ben definita con la ferma intenzione di servire il suo paese con la penna, come altri l'aveva servito con la spada. Non v'era più in lui come pungolo la vanità del giovinetto che chiede ai versi la fama, ma un'alta coscienza dei fini dell'arte e una virile ambizione di prendere il suo posto nella letteratura del suo tempo. (Gallarati Scotti 1934: 73)

Una dichiarazione analoga, dallo stesso sapore programmatico, viene inserita ex novo nell'edizione del ' 34 poche pagine dopo. Di seguito il raffronto con il corrispettivo brano del 1920 .

\begin{tabular}{|c|c|}
\hline Edizione del '20 & Edizione del '34 \\
\hline $\begin{array}{l}\text { Traspariva dalle sue prole la fiducia di poter } \\
\text { essere chiamato a compiere una missione nel } \\
\text { suo paese. E forse fin da allora guardandosi } \\
\text { intorno e considerando la poverta della } \\
\text { letteratura romantica gli parve di essere } \\
\text { predestinato a raccogliere una grande eredità } \\
\text { spirituale: quella del Manzoni }{ }^{33} \text {. Il } \\
\text { Fogazzaro era un temperamento morale e } \\
\text { intellettuale lontano dal Manzoni. }\end{array}$ & $\begin{array}{l}\text { Traspariva dalle sue prole la fiducia di poter } \\
\text { essere chiamato a compiere una missione nel } \\
\text { suo paese, liberato politicamente ma non } \\
\text { letterariamente dal giogo straniero. Nel } \\
\text { rinnovamento della vita italiana appena } \\
\text { iniziata egli sapeva ormai quale era il suo } \\
\text { compito. E forse fin da allora, guardandosi } \\
\text { intorno e considerando la povertà della } \\
\text { letteratura romantica gli parve di essere } \\
\text { predestinato a raccogliere una grande eredita } \\
\text { spirituale: quella del Manzoni. Un solco } \\
\text { profondo era stato aperto in questa terra } \\
\text { vergine dai Promessi Sposi. Bisognava } \\
\text { mettersi per quel solco non come un } \\
\text { imitatore della lettera, ma come un } \\
\text { continuatore dello spirito, non copiando } \\
\text { gli schemi esterni del capolavoro, come } \\
\text { avevano fatto i "manzoniani", ma } \\
\text { rivivendo secondo il proprio } \\
\text { temperamento lo spirito di un'arte } \\
\text { inimitabile. Il Fogazzaro era un } \\
\text { temperamento morale e intellettuale lontano } \\
\text { dal Manzoni. }\end{array}$ \\
\hline
\end{tabular}

Sull'originale romanzo Malombra l'operazione critica è piuttosto delicata e tale da suscitare più di una perplessità; lo stesso Danelon ritiene che Gallarati abbia sottovalutato il valore di Malombra e afferma espressamente «di trovare poco

${ }^{33}$ Circa l'accostamento, non semplice, tra Fogazzaro e Manzoni, cfr. Spera (2011: 459). 
condivisibili, se non sbagliate» le parole «assai riduttive, riservate a Malombra» (Danelon 2010: 106). Il romanzo però era nato nel periodo in cui Fogazzaro si era interessato all'occultismo, e perciò la valutazione dell'opera doveva necessariamente essere prudente proprio perché Gallarati imposta l'analisi dei romanzi come un'analisi dell'animo del loro autore. Dunque da un lato preferisce glissare su aspetti problematici, come appunto l'interesse per l'occultismo, e dall'altro sottolineare il valore del romanzo come tappa significativa di una storia dell'anima, anima dunque destinata ancora a maturare e crescere. Rendono ragione di questo doppio procedimento i due esempi che seguono:

\begin{tabular}{|c|c|}
\hline Edizione del '20 & Edizione del '34 \\
\hline --- & $\begin{array}{l}\text { Per questo tra la vita giovanile e il primo } \\
\text { romanzo del Fogazzaro vi è un nesso } \\
\text { profondo e sottile. L'uno serve a illuminare } \\
\text { l'altra. E studiando Silla, noi scopriamo un } \\
\text { Fogazzaro più appassionato e complesso di } \\
\text { quello che molti, anche dei suoi prossimi o } \\
\text { dei suoi più superficiali biografi, } \\
\text { sospettassero, ma un Fogazzaro } \\
\text { sincerissimo, che si confessa debole e } \\
\text { malato. Tanto che il libro ci appare come il } \\
\text { primo capitolo di una confessione, che } \\
\text { proseguirà e si approfondirà di romanzo in } \\
\text { romanzo. }\end{array}$ \\
\hline $\begin{array}{l}\text { Vi era stato un momento in cui il suo } \\
\text { pensiero aveva sentito il fascino di questa } \\
\text { soluzione al gran mistero della vita. Una } \\
\text { vaga religiosità che si soffermi con } \\
\text { insistenza sul problema della personalità } \\
\text { può condurre facilmente alla fede nelle } \\
\text { reincarnazioni, specialmente quando si è } \\
\text { come il Fogazzaro attenti scrutatori di } \\
\text { ogni moto di pena o di gioia che passa } \\
\text { nella propria vita interiore; quando si } \\
\text { tende lorecchio a ogni palpito lieve della } \\
\text { nostra anima che sembri il ricordo di un } \\
\text { mondo lontano; quando si ha così acuto il } \\
\text { senso del dolore e della giustizia da } \\
\text { rendere incomprensibili le sofferenze } \\
\text { immeritate nel mondo. Era stato un } \\
\text { periodo breve }\end{array}$ & $\begin{array}{l}\text { Vi era stato un momento in cui il suo } \\
\text { pensiero, vagando fuori dal cristianesimo, } \\
\text { aveva sentito il fascino di questa soluzione } \\
\text { al gran mistero della vita. Era stato un } \\
\text { periodo breve }\end{array}$ \\
\hline
\end{tabular}

Il Fogazzaro «sincerissimo, che si confessa debole e malato» dell'edizione del ' 34 basta a scusarlo di alcune divagazioni potenzialmente sospette di non ortodossia presenti in Malombra; nel secondo esempio invece è evidente come con una frase riassuntiva e piuttosto reticente («vagando fuori dal cristianesimo») Gallarati eviti di addentrarsi in meandri pericolosi che invece nel ' 20 affronta decisamente più di petto. 


\subsubsection{La corrispondenza con Elena}

Un punto delicato è la corrispondenza con Elena, il nome fittizio dato a una donna che tenne con Fogazzaro uno scambio epistolare fitto, perlopiù imperniato su tematiche religiose ma dai toni molto intimi. Modellato su questa donna reale, nasce il personaggio femminile di Elena, la protagonista insieme a Daniele del romanzo Daniele Cortis. Un romanzo esaltato da Gallarati perché il protagonista vi figura quale intellettuale politicamente impegnato, capace di un amore fortissimo e al contempo concretamente attivo nella realtà sociale. È su questo aspetto che Gallarati ritiene opportuno insistere e per questo l'edizione del '34 vede l'aggiunta, qui e là, di brevi brani che documentano in dettaglio la realtà politica di quel periodo, o di citazioni da quelle che Gallarati chiama Brevi note politiche inedite di Fogazzaro. Tuttavia, rispetto all'edizione del '20, si cerca anche, pur valorizzandolo, di dare al messaggio politico del Daniele Cortis una distanza storica, grazie a un certo distacco e a un ridimensionamento del pensiero di Fogazzaro al periodo in cui l'autore visse e lo formulò. Ecco due esempi in proposito.

\begin{tabular}{|c|c|}
\hline Edizione del '20 & Edizione del '34 \\
\hline $\begin{array}{l}\text { è necessario rimetterci } p \\
\text { vita italiana di quel perio }\end{array}$ & $\begin{array}{l}\text { è necessario rimetterci per un momento nella } \\
\text { vita italiana di quel periodo e vedere quale } \\
\text { intimo nesso ricolleghi le idee del Cortis } \\
\text { all'età che fu sua. }\end{array}$ \\
\hline $\begin{array}{l}\text { Noi siamo i primi a vedere quanto facile } \\
\text { sarebbe, oggi, la critica a molte idee } \\
\text { politiche del Daniele Cortis, specialmente } \\
\text { in rapporto allo sviluppo della società } \\
\text { democratica, ch'egli pensava come una } \\
\text { redenzione del proletariato per l'opera } \\
\text { filantropica dello Stato, non come una } \\
\text { partecipazione autonoma delle classi } \\
\text { lavoratrici alla formazione del nuovo } \\
\text { diritto. Ma considerato come una } \\
\text { reazione contro lo scetticismo e l'inerzia } \\
\text { del suo tempo, esso continua a avere il } \\
\text { valore di una battaglia non invano } \\
\text { combattuta. }\end{array}$ & $\begin{array}{l}\text { Noi siamo i primi a vedere quanto facile } \\
\text { sarebbe, oggi, la critica a molte idee } \\
\text { politiche del Daniele Cortis. Ma avendo } \\
\text { avuto una lunga e intima consuetudine } \\
\text { con lui, e avendo tra le mani molte } \\
\text { centinaia di lettere sue e vagliato sicure } \\
\text { testimonianze, possiamo riaffermare con } \\
\text { piena coscienza che ogni altra } \\
\text { interpretazione del suo pensiero politico, } \\
\text { in base a convinzioni o atteggiamenti o } \\
\text { opportunità posteriori all'età che fu sua, suesti } \\
\text { sono come in genere tutti questi } \\
\text { travisamenti polemici privi di onestà } \\
\text { storica. Nel romanzo del Fogazzaro, noi, } \\
\text { invece, non abbiamo inteso di compiacerci } \\
\text { a ritrovare idee che ci sono care, ma a } \\
\text { chiarire secondo verità quelle di un uomo } \\
\text { che è nato nel 1842 ed è morto nel 1911. }\end{array}$ \\
\hline
\end{tabular}

In entrambi i brani riportati è chiaro il tentativo di circoscrivere le posizioni presenti nel romanzo di Fogazzaro all' «età che fu sua».

La figura di Elena invece, esaltata nell'edizione del '20 e del '34, viene ridimensionata dal giudizio critico dell'ultima edizione, nel ' 63 , che prevede una sorta di inversione, per cui la figura di Daniele viene vista più positivamente rispetto a quella di Elena, come mostrano gli esempi qui riportati. 


\begin{tabular}{|c|c|}
\hline Edizione del '34 & Edizione del '63 \\
\hline $\begin{array}{l}\text { Eppure per quanto interessante sia il Daniele } \\
\text { Cortis studiato in questi suoi aspetti interiori } \\
\text { e nel suo lato politico, esso apparterrebbe } \\
\text { già alla storia, se non vivesse, come opera } \\
\text { d'arte, per una figura che tra ciò che nel } \\
\text { libro è caduco ha la giovinezza eterna della } \\
\text { poesia: Elena. Perchè essa - la più amata - } \\
\text { e anche la più perfetta tra le creature nate } \\
\text { dal cuore di Antonio Fogazzaro. Le altre } \\
\text { donne della sua opera letteraria al confronto } \\
\text { impallidiscono tutte. }\end{array}$ & $\begin{array}{l}\text { Eppure per quanto interessante sia il Daniele } \\
\text { Cortis studiato in questi suoi aspetti interiori } \\
\text { e nel suo lato politico, esso apparterrebbe } \\
\text { già alla storia, se non vivesse, come opera } \\
\text { d'arte, per una figura che tra ciò che nel } \\
\text { libro è caduco ha la giovinezza persistente } \\
\text { della poesia: Elena. Le altre donne amanti } \\
\text { della sua opera letteraria al confronto } \\
\text { impallidiscono tutte. }\end{array}$ \\
\hline $\begin{array}{l}\text { Daniele Cortis è, da un punto di vista } \\
\text { puramente artistico, la parte morta e } \\
\text { pesante del libro. }\end{array}$ & $\begin{array}{l}\text { Daniele Cortis è, da un punto di vista } \\
\text { puramente artistico, la parte voluta e } \\
\text { pensante del libro. }\end{array}$ \\
\hline $\begin{array}{l}\text { Questa donna, contenuta nel suo dolore, } \\
\text { quasi senza gesti nella passione, si volgerà } \\
\text { ancora ai lontani dal suo esilio di oltremare, } \\
\text { per rivelare ciò che vi fu di più nascosta } \\
\text { poesia e di più chiuso ardore nella } \\
\text { tormentata anima femminile dei nostri } \\
\text { giorni, ciò che sfuggì, di spirituale passione, } \\
\text { ai letterati e che era oltre la letteratura. }\end{array}$ & - \\
\hline
\end{tabular}

Nei primi due casi, il giudizio sulla figura femminile del romanzo viene un poco stemperato in favore di una maggiore enfasi posta sul personaggio maschile (sebbene Daniele sia comunque, sin dall'edizione del ' 20 , considerato un carattere molto ben riuscito). L'ultima frase invece, che conclude il capitolo settimo nell'edizione del '34, con una sorta di esaltazione oltre il romanzo e oltre il tempo della figura di Elena, viene del tutto eliminata nell'edizione del '63. Questa esaltazione oltre il tempo era ancora più marcata nell'edizione del ' 20 , che chiudeva la frase con l'aggiunta di un sintagma: «[...] oltre la letteratura, nel cuor della vita» (Gallarati Scotti 2011: 138).

Dei capitoli successivi al Daniele Cortis, e prima di quello dedicato a Piccolo Mondo Antico, merita attenzione il capitolo intitolato Scienza e fede, come esempio del puntuale intervento di correzione di posizioni inaccettabili per 1'ortodossia religiosa, che Gallarati provvede, punto per punto, a smussare. Questo capitolo, nell'edizione del '20, diceva tutto 1'entusiasmo con cui Fogazzaro aveva accolto le teorie darwiniane e come le aveva inglobate nel suo sentimento religioso. Si toccavano però alcuni punti particolarmente delicati sui quali l'intervento autocensorio si rese necessario. La reticenza dell'edizione del '34, pur non negando recisamente quanto scritto nel ' 20 , evita accuratamente di addentrarsi in dettagli compromettenti. Eccone qualche esempio. 


\begin{abstract}
Edizione del '20
al tempo in cui Fogazzaro cominciò a occuparsi del problema dell'evoluzione la parola "darwinismo" significava [...] una dottrina della vita tendente a abbattere la millenaria dottrina del cristianesimo [...]. D'altra parte a questo "scientismo" dogmatico si opponeva nel campo cattolico uno spirito timoroso, ostile alla ricerca piena della verità. Una crisi di coscienza pareva minacciare i credenti che confrontavano la lettera del racconto biblico con la Storia della Creazione quale la venivano narrando i fossili al geologo e gli animali al biologo. Nonostante la larga visione di S. Agostino, la lettera aveva ancora nella Chiesa un valore indiscusso ed era custodita gelosamente così come era stata trasmessa dalla Sinagoga. La gretta ortodossia non era tuttavia la sola che sentisse l'urto formidabile dei nuovi progressi scientifici.
\end{abstract}

Noi oggi possiamo criticare facilmente i Noi oggi possiamo criticare facilmente i discorsi del Fogazzaro - e lo faremo - [...]. discorsi del Fogazzaro [...]. Ma ciò che non Ma ciò che non è invecchiato in quegli studi, è invecchiato in quegli studi è il sentimento è il sentimento mistico con cui li intraprese religioso con cui li intraprese e che li e che li informa tutti, il fervore nuovo con informa tutti, il fervore nuovo con cui cui cercava Iddio tra i materialisti e i cercava Iddio.

teologi del suo tempo, egli poeta e credente a cui si era presentata una nuova visione della vita e che sentiva intorno a sé con un brivido sacro, non più un piccolo mondo ben plasmato e fissato da un Creatore esterno e immobile nel suo cielo, ma un universo attraversato da correnti di vita perenne, una creazione senza limiti di tempo e di spazio.

Guardati dal punto di vista della scienza, Guardati dal punto di vista della scienza, questi discorsi non dicono nulla di originale. questi discorsi non dicono nulla di originale. Ma nella storia del sentimento religioso in Ma nella storia del sentimento religioso in Italia essi rimangono come un documento Italia essi rimangono come un documento importante del passo decisivo fatto dalla importante. Segnano l'ora in cui uno spirito coscienza mistica, fuori dal di poeta senti, nel cattolicismo, l'azione tradizionalismo letterale che l'opprimeva. divina.

Segnano l'ora in cui uno spirito precursore

sentì, nel cattolicismo, l'azione divina...

Ma se era disposto a ascoltare le giuste Ma se era disposto a ascoltare le giuste critiche $[\ldots]$ non era disposto in nessun critiche [...] non era disposto in nessun modo a abbandonare la sua idea modo a abbandonare la sua idea fondamentale. Era convinto di essere nel fondamentale.

vero. A una condanna della Chiesa non si sarebbe ribellato; ma non avrebbe per essa sconfessato le idee di cui era persuaso. 
L'intento è chiarissimo. Gallarati rientra nei panni di critico ed evita di addentrarsi in terreni che implicano un giudizio sull'operato e sul pensiero della Chiesa: lascia da parte considerazioni sulla «crisi di coscienza» prodotta dalla «gretta ortodossia» (primo esempio); evita di contrapporre il Fogazzaro «credente» ai «materialisti e teologi del suo tempo» (secondo esempio); precisa che Fogazzaro resta un «poeta», con ciò chiamandolo fuori da una responsabilità maggiore che gli verrebbe dall'essere definito come «spirito precursore» di nuove idee (terzo esempio). Sull'ultimo brano in particolare si era soffermato nel suo Voto anche padre Rosa: «la proposizione è scandalosa, specialmente in quanto attribuita a chi viene lodato come uno dei migliori cattolici del suo secolo; alias damnata nel silenzio ossequioso dei giansenisti e nella ostinazione che loda, è proxima haeresi» (Zambarbieri 2013: 35). L'unica soluzione, in questi casi, è la reticenza: Gallarati non può certo provarsi a confutare le posizioni religiose ortodosse, e preferisce non condannare direttamente le idee di Fogazzaro o tentare (e sarebbe stata impresa assai complessa) di giustificarle; pertanto preferisce più cautamente tagliare.

\subsubsection{Piccolo Mondo Antico}

Quanto a Piccolo Mondo Antico, come si è detto, la valutazione di quest'opera come il vertice della produzione fogazzariana rimane costante dalla prima alla seconda, fino alla terza edizione. Un esempio significativo è il brano che segue, dove si può vedere, nel passaggio da un'edizione all'altra, l'enfasi crescente sul valore dell'opera che consacra definitivamente Fogazzaro tra i "classici" della letteratura italiana.

\begin{tabular}{|c|c|}
\hline Edizione del '20 & Edizione del '34 \\
\hline $\begin{array}{l}\text { Eppure non credo di ingannarmi } \\
\text { affermando che il valore di Piccolo } \\
\text { Mondo Antico è sufficiente a porre il } \\
\text { Fogazzaro come il continuatore di } \\
\text { una tradizione nazionale cristiana } \\
\text { nell'arte del nostro tempo. }\end{array}$ & $\begin{array}{l}\text { Opere che furono chiamate immortali non } \\
\text { ebbero che una rinomanza effimera; e spesso chi } \\
\text { scrive solo con l'illusione di prolungare il suono } \\
\text { del proprio nome oltre la fossa somiglia a quel } \\
\text { bambino che costruisce una statua di arena sulla } \\
\text { spiaggia del mare e non pensa all'onda che la } \\
\text { spazzerà via nella notte. Vi è una tacita, } \\
\text { invisibile onda che lava la terra anche dalla } \\
\text { letteratura quotidiana e la lascia a ogni alba di } \\
\text { secolo quasi nuda. Poche cose resistono: qualche } \\
\text { blocco di granito, qualche statua di bronzo. } \\
\text { Questo pensiero ci deve rendere umili come } \\
\text { scrittori e cauti come critici. } \\
\text { Ma so di non essermi ingannato - e la critica di } \\
\text { questi ultimi anni non ha fatto che riconfermare } \\
\text { e mettere in luce le mie conclusioni anche } \\
\text { quando è sembrato che facessi una nuova } \\
\text { scoperta - nel proclamare che il valore di Piccolo } \\
\text { Mondo Antico e sufficiente per dare al Fogazzaro } \\
\text { un primissimo posto tra i grandi scrittori italiani } \\
\text { del secolo XIX e che il suo romanzo fa parte del } \\
\text { più puro patrimonio letterario nazionale. }\end{array}$ \\
\hline
\end{tabular}


Canonizzare Fogazzaro tra gli autori classici italiani significa anche fare della scelte. Per questo nel ' 34 si inserisce un giudizio abbastanza netto sulla poesia del Carducci, in opposizione alla poesia religiosa impegnata di Dante e Manzoni, con lo scopo evidente di collocare Fogazzaro dalla parte di questi ultimi in opposizione al primo. Gallarati scrive infatti nel ' 34 che Carducci

rimane l'esponente glorioso di un atteggiamento di rivendicazioni civili, patriottiche, di rivolte umanistiche, ma non è il genio, né la voce intera di un popolo che non si riallaccia solo a Roma imperiale nelle sue tradizioni, ma a tutto lo sviluppo del cristianesimo, e che ha dato al mondo Dante e Manzoni (Gallarati Scotti 1934: 295).

Dal capitolo tredicesimo ( Cattolico e liberale» ${ }^{34}$ ) in poi, i mutamenti dalla prima alla seconda edizione consistono sostanzialmente nell'eliminare affermazioni, giudizi, valutazioni e commenti che riguardano temi religiosi. Gli unici inserimenti nuovi sono sostanzialmente composti di materiale documentario, mentre tutto ciò che costituisce il commento di Gallarati alle posizioni di Fogazzaro (specialmente sulla sua vicinanza al movimento del Modernismo) e alle sue ultime opere (soprattutto Il Santo) è oggetto di massiccio sfrondamento.

Moltissimi passi si potrebbero citare, poiché le correzioni sono veramente fitte. Un esempio per tutti può essere il ridimensionamento della figura di Rosmini, tanto lodato nell'edizione del '20, di cui invece si evitano elogi diretti e soprattutto giudizi di merito nell'edizione del '34.

\begin{tabular}{|c|c|}
\hline Edizione del '20 & Edizione del '34 \\
\hline $\begin{array}{l}\text { D'altra parte è nel Rosmini ch'egli } \\
\text { giustificò di fronte a sé stesso quella sua } \\
\text { intuizione poetica della animazione della } \\
\text { natura che lo aveva condotto dai sogni } \\
\text { dell'adolescenza } \\
\text { sull'evoluzione }{ }^{35} \text {. agli }\end{array}$ & $\begin{array}{l}\text { D'altra parte gli sembrava di trovare in } \\
\text { Rosmini anche una guida filosofica per i } \\
\text { suoi studi sull'Evoluzione. }\end{array}$ \\
\hline
\end{tabular}

$\mathrm{O}$ ancora si pensi al giudizio sul papa Pio X (e implicitamente su movimenti come quello modernista) e anche la condivisione da parte di Fogazzaro delle tesi dell'abate Loisy (le cui opere vennero messe all'Indice per Modernismo).

34 Nell'edizione del '34 e del '63 il titolo del capitolo cambia in «Nel solco del Risorgimento».

${ }^{35}$ Padre Rosa giudica questa espressione come «falsa et erronea, et alias damnata» (Zambarbieri 2013: 36). 


\begin{tabular}{|l|l|}
\hline Edizione del '20 & Edizione del '34 \\
\hline [Fogazzaro] Come cattolico si inchinava alla & Come cattolico si inchinava alla Volontà le \\
Volontà le cui vie ci sono oscure. Pensava & cui vie ci sono oscure e la cui \\
che ogni avvenimento storico e la & imperscrutabile logica si rivela solo \\
nomina del visibile capo della Chiesa in & lentamente nella storia della Chiesa. \\
modo particolare - ha certo un valore di & \\
atto provvidenziale nella imperscrutabile & \\
logica divina. Ma sentiva in pari tempo \\
che nella Chiesa militante non tutta la \\
vita religiosa può riassumersi nelle \\
opinioni e nei metodi del papa; che molte \\
altre correnti, purché disciplinate, è bene \\
che si manifestino nella viva compagine \\
del cattolicismo e che una certa religiosa \\
opposizione al governo chiesastico non \\
solo è lecita, ma spesso è santa come ne fa \\
fede la storia che ne constata l'azione \\
perenne attraverso ai secoli. \\
$\begin{array}{l}\text { Ora in questo senso egli si sentì, fin dal } \\
\text { primo momento, dalla parte }\end{array}$ \\
dell'opposizione
\end{tabular}

Sostenere, come si dice nel primo brano, che «non tutta la vita religiosa può riassumersi nelle opinioni e nei metodi del papa» (qui papa Pio X) e che quindi «una certa religiosa opposizione al governo chiesastico [...] spesso è santa» è affermazione troppo netta per essere smussata, e troppo forte per essere lasciata; Gallarati perciò decide di tagliare. Quando poi non è possibile dichiarare Fogazzaro estraneo a certe posizioni, se non altro Gallarati cerca di sminuirne eventuali responsabilità con l'attenuante dell'ingenuità e dell'entusiasmo. Allo scopo, descrive Fogazzaro quasi come la vittima di un raggiro, cioè tra quelli «che si lasciarono facilmente prendere dalla parte più esteriore eingannatrice dell'opera delLoisy»».

\subsubsection{Il Santo}

Per quanto riguarda Il Santo, le correzioni di Gallarati prediligono ancora una volta l'attenuazione dei contrasti. Non potendo infatti (e soprattutto non volendo) smentire la tesi di un Fogazzaro intellettuale impegnato, o di un Fogazzaro che si rispecchia nelle sue opere, pena la confutazione della base su cui poggia l'intera

${ }^{36}$ Per padre Rosa la frase è «ancora più iniuriosa Ecclesiae et eius praxi, male sonans» (Zambarbieri 2013: 36). 
Vita, Gallarati tenta di stemperare la responsabilità di Fogazzaro attribuendo sostanzialmente ad una sorta di generico "sentimento poetico" quella che nell'edizione del ' 20 descriveva come una programmatica denuncia delle storture del mondo ecclesiastico (a tutto vantaggio, per contro, di coloro che di tali storture si facevano espliciti accusatori). Anche in questo caso gli esempi potrebbero essere molti; in alcuni casi Gallarati decide di tagliare completamente brani lunghi qualche pagina, relativi ad illustrare quale fosse il tipo di rinnovamento che Fogazzaro auspicava nella Chiesa (Gallarati Scotti 2011: 342-345) e come egli denunciasse, nel dualismo laicato-clero, l'ipertrofia del potere del clero (Gallarati Scotti 2011: 345-346). In altri casi Gallarati interviene modificando o espungendo brevi sezioni; basti qui qualche esempio, tra $i$ tanti, per dare l'idea della prova di equilibrismo data da Gallarati su questa parte, che può davvero considerarsi la più delicata dell'intera Vita.

Talvolta Gallarati dunque vuole dare del Fogazzaro un'idea di autore appassionato, che in maniera forse incauta (e per questo - concede Gallarati - in parte sanzionabile) ma comunque non colpevole, si addentra in ambiti che non sono i suoi, come nei casi seguenti.

\begin{tabular}{|c|c|}
\hline Edizione del '20 & Edizione del '34 \\
\hline $\begin{array}{l}\text { [il romanzo Il Santo] diventò come il } \\
\text { rifugio di tutte quelle aspirazioni, che } \\
\text { nella realtà erano frustrate; l'espressione } \\
\text { di una corrente di rinnovamento mistico } \\
\text { che doveva fatalmente urtare contro la } \\
\text { rigida difesa teologica di cui Pio X doveva } \\
\text { fare il fulcro del suo regno. }\end{array}$ & $\begin{array}{l}\text { Quasi inconsciamente egli si trovò ad } \\
\text { assumere nel romanzo una posizione di } \\
\text { battaglia e di opposizione contro la } \\
\text { tendenza che prevaleva a fare del suo } \\
\text { personaggio un antesignano di idee che } \\
\text { dovevano purtroppo sboccare in un } \\
\text { conflitto con le direttive di Pio } X \text {. }\end{array}$ \\
\hline $\begin{array}{l}\text { Il Fogazzaro ha resistito alla tentazione } \\
\text { delle soluzioni facili. Ha voluto porre il suo } \\
\text { Santo di fronte ai problemi più tormentosi e } \\
\text { alle difficoltà più gravi. }\end{array}$ & $\begin{array}{l}\text { o ha voluto invece porre il suo } \\
\text { onte ai problemi più tormentosi e } \\
\text { tà più gravi per cui la sua arte } \\
\text { ta. }\end{array}$ \\
\hline $\begin{array}{l}\text { Non credo inutile perciò di riesaminare }[\ldots] \\
\text { l'ispirazione religiosa del Santo, } \\
\text { rispondendo senza spirito polemico a gruppi } \\
\text { di oppositori, a gruppi di inquisitori, non } \\
\text { come chi vuol difendere, ma come chi vuol } \\
\text { chiarire. }\end{array}$ & $\begin{array}{l}\text { Non credo inutile perciò di riesaminare }[\ldots] \\
\text { l'ispirazione religiosa del Santo, } \\
\text { rispondendo senza spirito polemico non già } \\
\text { alla autorità della Chiesa, di cui } \\
\text { accettiamo senza discussione la condanna, } \\
\text { ma a gruppi di oppositori, non come chi } \\
\text { vuol difendere, ma come chi vuol chiarire. }\end{array}$ \\
\hline
\end{tabular}

Si noti, nel primo esempio, l'avverbio «inconsciamente» che quasi mette Fogazzaro al riparo da se stesso e da una presa di posizione pensata e voluta (e quindi a maggior ragione responsabile); nel secondo esempio c'è poi un chiaro ridimensionamento dell'ambito di pertinenza di Fogazzaro, la cui colpa, al limite, è quella di avere incautamente affrontato tematiche per le quali non aveva strumenti adeguati e «per cui la sua arte non era fatta». Nel terzo esempio poi, l'esplicita accettazione «senza discussione» della sanzione ecclesiastica vuole rassicurare circa la valutazione generale dell'opera di Fogazzaro.

Talvolta invece, quando la frase originaria è troppo forte da renderne impossibile 
una limatura, Gallarati preferisce tagliare, come nei casi che seguono.

\begin{tabular}{|c|c|}
\hline Edizione del '20 & Edizione del '34 \\
\hline $\begin{array}{l}\text { [Il Santo] Era un libro che gli veniva dai } \\
\text { profondi dell'anima, era una aspirazione di } \\
\text { tutta la sua vita, la sintesi di tutte le sue } \\
\text { esperienze religiose. }\end{array}$ & -- \\
\hline $\begin{array}{l}\text { La sua arte sarebbe entrata là dove la sua } \\
\text { persona era esclusa. Questo pensiero lo } \\
\text { esaltava e dava ai suoi occhi un valore } \\
\text { nuovo a ciò che scriveva. Non lavorava } \\
\text { per la gloria letteraria ma per una } \\
\text { missione religiosa. }\end{array}$ & $\begin{array}{l}\text { La sua arte sarebbe entrata, almeno sperava } \\
\text { così, là dove la sua persona era esclusa. }\end{array}$ \\
\hline $\begin{array}{l}\text { Perciò è giusto che il Santo sia stato } \\
\text { considerato dai suoi nemici più che come } \\
\text { una opera di letteratura, un programma } \\
\text { di riforma cattolica. Voleva essere una } \\
\text { battaglia e lo fu. Né sarebbe onesto } \\
\text { nasconderlo oggi, criticando il libro dal solo } \\
\text { punto di vista dell'arte e rimproverando ai } \\
\text { suoi oppositori di aver condotto senza } \\
\text { ragione la guerra sul campo del pensiero } \\
\text { religioso dell'autore. Noi anzi crediamo } \\
\text { che il valore di questo romanzo sta } \\
\text { nell'essere una azione. }\end{array}$ & $\begin{array}{l}\text { Voleva essere una battaglia e lo fu. Né } \\
\text { sarebbe onesto nasconderlo oggi, criticando } \\
\text { il libro dal solo punto di vista dell'arte e } \\
\text { rimproverando ai suoi oppositori di aver } \\
\text { condotto senza ragione la guerra sul campo } \\
\text { del pensiero religioso dell'autore. }\end{array}$ \\
\hline
\end{tabular}

Come attenuare l'idea che Il Santo fosse «una aspirazione di tutta la sua vita» (primo esempio), o che Fogazzaro con quest'opera «non lavorava per la gloria letteraria ma per una missione religiosa» (secondo esempio), o addirittura come aggiustare quel commento che riteneva «giusto» che Il Santo fosse considerato «più che come una opera di letteratura, un programma di riforma cattolica» (terzo esempio)? Correggere, smussare, attenuare qui non si poteva e, non volendo negare, Gallarati sostanzialmente preferisce tacere.

\subsection{Altri aspetti della Vita}

Il procedimento di Gallarati resta il medesimo nei restanti capitoli della Vita. In particolare il capitolo «La parola di Don Giuseppe Flores» affronta la questione dell'enciclica Pascendi, che condannava apertamente il Modernismo. Anche in questo caso molto ampi sono i brani stralciati per intero, nei quali, almeno in parte, si sottolineava l'eccessiva durezza dell'enciclica ${ }^{37}$. Le ultime pagine, infine, sono dedicate agli ultimi momenti di sofferenza e di solitudine di Fogazzaro, al suo

37 Ciò vale per il lungo capoverso di pp. 390-391, dove Gallarati spiega che nell'enciclica vi è qualcosa di «ingiustamente aggressivo, di schematicamente inesatto, di puramente negativo e di praticamente inconciliabile col pensiero e con la vita del nostro tempo» (Gallarati Scotti 2011: 390). 
ripiegamento su se stesso, alla stesura dell'ultimo sofferto romanzo, Leila (anch'esso colpito dalla censura ecclesiastica), e a un bilancio conclusivo della sua vita di uomo, di cristiano e di scrittore. Nell'ultimo capitolo si segnala qualche correzione tra la seconda e la terza edizione un po' più significativa rispetto a quelle, quasi tutte minime, presenti qua e là nel resto della Vita. Queste ultime correzioni vanno nella direzione di un bilancio finale sulla figura di Fogazzaro, e tendono, da una certa distanza, a superare la necessità di talune puntualizzazioni riguardanti la polemica religiosa (tanto urgenti in occasione della prima e della seconda edizione, quanto superate poi) e soprattutto a dare del Fogazzaro un'immagine, per quanto piena di ricordo riconoscente e affettuoso, meno eroica, e più semplicemente e dolcemente disegnata sull'uomo acuto e autore sensibile che Gallarati tiene a descrivere. Si vedano, a titolo d'esempio, i brani che seguono, tutti espunti dall'edizione del ' 63 .

\begin{tabular}{|l|l|}
\hline Edizione del '34 & Edizione del '63 \\
\hline $\begin{array}{l}\text { Ma io mi accorgo con meraviglia che vi è } \\
\text { un lato di lui che è ancora attuale, che parla }\end{array}$ & $\begin{array}{l}\text { Ma tuttavia vi è un lato che è ancora } \\
\text { attuale, che parla ancora alle anime [...] e le }\end{array}$ \\
$\begin{array}{l}\text { ancora alle anime [...] e le consola; che vi è } \\
\text { una ripresa di interesse per la sua vita e i }\end{array}$ & consola. \\
suoi libri, anche tra i giovani, e un \\
riaccendersi di polemiche letterarie \\
intorno al suo nome che non si \\
spiegherebbero se egli non dominasse \\
ancora su molti spiriti.
\end{tabular}




\section{PRIME CONCLUSIONI}

Il raffronto tra le diverse edizioni permette dunque di cogliere insieme un cambiamento di prospettiva e una coerenza di fondo; un recupero critico di quanto pensato sin dall'inizio (si pensi alle parti del manoscritto originario che tornano nella seconda edizione) ma insieme una correzione (non inversione!) di rotta.

Nel 1904 Gallarati pubblicava, sul Secolo XX, Il poeta dell'ideale, un articolo dedicato a Fogazzaro. Riconsiderando le parole di questo titolo, la Vita di Antonio Fogazzaro scritta subito dopo la sua morte ha tutto il valore dell'affettuosa celebrazione dell'amico come "poeta ideale" se non addirittura come "cristiano ideale", senza preposizioni. L'irruenza, l'entusiasmo, l'incisività dei commenti di Gallarati Scotti sono poi costretti a cedere il passo a uno sguardo più prudente negli anni successivi, sia per un maggiore distacco dalle vicende di cui Gallarati non nasconde - anzi rivendica, almeno nella prima edizione - di aver fatto parte, sia per necessità, vista la condanna ecclesiastica che colpì l'edizione del '20. Fogazzaro di conseguenza, dalla seconda edizione della Vita in poi, viene definitivamente descritto non pensatore ideale, ma più umanamente, e con accenti che rimangono sin dalla prima edizione commossi e quasi lirici, celebrato come poeta dell'ideale.

\section{RIFERIMENTI BIBLIOGRAFICI}

DANELON, Fabio (2010): «"Un solitario delle lettere”? Formazione, prime esperienze scrittorie e rapporto con Antonio Fogazzaro di Tommaso Gallarati Scotti», in Luciano Pazzaglia (a cura di), Tommaso Gallarati Scotti e il suo tempo. Dalla crisi modernista all'interventismo democratico, Milano, Unicopli, pp. 91-109.

FoGAZZARO, Antonio (1940): Lettere scelte, a cura di Tommaso Gallarati Scotti, Milano, Mondadori.

CrevennA, Claudia (2011): «Nota alle tre edizioni della Vita», in Tommaso Gallarati Scotti, La vita di Antonio Fogazzaro, a cura di C. Crevenna, Brescia, Morcelliana, pp. 471-490.

CRISPOLTI, Filippo (1920): «A. Fogazzaro nella Vita di T. Gallarati-Scotti», Nuova Antologia, serie 6, vol. CCVI della raccolta CCXC, pp. 120-142.

GALlARATI SCOTTI, Tommaso (1904): «Il poeta dell'ideale: Antonio Fogazzaro», Il Secolo XX, anno 3, n. IV, pp. 267-287.

Gallarati ScOTTI, Tommaso (1920): La vita di Antonio Fogazzaro, Milano, Baldini e Castoldi.

Gallarati SCOTTI, Tommaso (1934): La vita di Antonio Fogazzaro. Dalle memorie e dai carteggi inediti, Milano, Mondadori.

Gallarati SCOTTI, Tommaso (1963): La vita di Antonio Fogazzaro. Dalle memorie e dai carteggi inediti, Milano, Mondadori. 
Gallarati SCOTTI, Tommaso (1982): La vita di Antonio Fogazzaro. Dalle memorie e dai carteggi inediti, a cura di Carlo A. Madrignani, Milano, Mondadori.

Gallarati ScotTI, Tommaso (2011): La vita di Antonio Fogazzaro, a cura di Claudia Crevenna, Brescia, Morcelliana.

GENTILE, Giovanni (1920): «La vita di Antonio Fogazzaro», La Critica-18,pp. 291-297.

GUASCO, Maurilio (2011): «Gallarati Scotti. Per una nuova spiritualità del laicato», in Tommaso Gallarati Scotti, La vita di Antonio Fogazzaro, a cura di Claudia Crevenna, Brescia, Morcelliana, pp. 439-453.

MADRIGNANI, Carlo Alberto (1982): «Introduzione», in Tommaso Gallarati Scotti, La vita di Antonio Fogazzaro. Dalle memorie e dai carteggi inediti, Milano, Mondadori, pp. V-XII.

MATTESINI, Francesco (1994): «La prima edizione della Vita di Antonio Fogazzaro (con lettere inedite)», in: Fulvio De Giorgi / Nicola Raponi (a cura di), Rinnovamento religioso e impegno civile in Tommaso Gallarati Scotti: atti del Colloquio nel centenario della nascita, Milano, Vita e pensiero, pp. 213-217.

MARCORA, Carlo (1984): L'accoglienza della biografia fogazzariana di Gallarati Scotti in ambiente ecclesiastico, in: Attilio Agnoletto / Enzo Noè Girardi / Carlo Marcora (a cura di), Antonio Fogazzaro, Milano, Franco Angeli, pp. 282-326.

MisCIATTELLI, Piero (1920): «La coscienza religiosa di Antonio Fogazzaro», Nuova Antologia, serie 6, vol. CCVI della raccolta CCXC, pp. 254-268.

RAPONI, Nicola (1972): «Tommaso Gallarati Scotti: appunti per una biografia», in Alessandro Pellegrini (a cura di), Tre cattolici liberali. Alessandro Casati, Tommaso Gallarati Scotti, Stefano Jacini, Milano, Adelphi, pp. 61-120.

RosA, Enrico (1920): «La Vita di Antonio Fogazzaro secondo Tommaso Gallarati Scottì, La civiltà cattolica, vol. III, anno 71, pp. 64-72.

SPERA, Francesco (2011): "Gallarati biografo e interprete di Fogazzaro», in Tommaso Gallarati Scotti, La vita di Antonio Fogazzaro, a cura di Claudia Crevenna, Brescia, Morcelliana, pp. 455-470.

ViolA, Gianni Eugenio (1994): «Il Fogazzaro di Gallarati Scotti», in: Fernando Bandini / Fabio Finotti (a cura di), Antonio Fogazzaro: le opere e i tempi. Atti del Convegno internazionale di studio. Vicenza, 27-28-29 aprile 1992, Vicenza, Accademia Olimpica, pp. 367-384.

ZAMBARBIERI, Annibale (2013): «La Vita di Antonio Fogazzaro all'Indice», in Luciano Pazzaglia / Claudia Crevenna (a cura di), Tommaso Gallarati Scotti tra totalitarismo fascista e ripresa della vita democratica, Milano, Cisalpino, pp. 13-40. 